\title{
Perspectives of patients, family caregivers and physicians about the use of opioids for refractory dyspnea in advanced chronic obstructive pulmonary disease
}

\author{
Graeme Rocker MHSc DM, Joanne Young RRT, Margaret Donahue MDiv, Morag Farquhar PhD, \\ Catherine Simpson MDiv
}

\begin{abstract}
Background: A recent national practice guideline recommends the use of opioids for the treatment of refractory dyspnea in patients with advanced chronic obstructive pulmonary disease (COPD). We conducted two qualitative studies to explore the experiences of patients and family caregivers with opioids for refractory COPD-related dyspnea and the perspectives and attitudes of physicians toward opioids in this context.
\end{abstract}

Methods: Patients ( $n=8 ; 5$ men, 3 women), their caregivers ( $n=12 ; 5$ men, 7 women) and physicians ( $n=28,17$ men, 11 women) in Nova Scotia participated in the studies. Semistructured interviews were recorded, transcribed verbatim, coded conceptually and analyzed for emergent themes using interpretive description methodology.

Results: Patients reported that opioids provided a sense of calm and relief from severe dyspnea.
Family caregivers felt that opioids helped patients to breathe more "normally," observed improvements in patients' symptoms of anxiety and depression, and experienced reductions in their own stress. Patients reported substantial improvements in their quality of life. All patients and family caregivers wanted opioid therapy to continue. Most physicians were reluctant to prescribe opioids for refractory dyspnea, describing a lack of related knowledge and experience, and fears related to the potential adverse effects and legal censure.

Interpretation: Discrepancies between the positive experiences of patients and family caregivers with opioids and the reluctance of physicians to prescribe opioids for refractory dyspnea constitute an important gap in care. Bridging this gap will require initiatives to improve the uptake of practice guidelines and to increase confidence in prescribing opioids for dyspnea refractory to conventional treatment.
Competing interests: None declared.

This article has been peer reviewed.

Correspondence to:

Dr. Graeme Rocker, graeme.rocker@dal.ca

CMAJ 2012. DOI:10.1503 /cmaj.111758
$\mathrm{T}$ he prevalence of chronic obstructive pulmonary disease (COPD) in Canada is high and rising. ${ }^{1}$ Dyspnea is the predominant symptom and, for patients with advanced COPD, conventional optimized treatments often fail to provide relief. ${ }^{2}$ Control of dyspnea can be an ongoing struggle. Many patients experience severe and unpredictable "dyspnea crises" that, when superimposed on relentless background dyspnea, often trigger additional fear, panic, feelings of helplessness and further erosion of quality of life among patients and their family caregivers. For those living with advanced COPD, symptom relief is important, ${ }^{3,4}$ and responding to their needs should be a priority. ${ }^{5}$

Opioids have long been used to relieve dyspnea in patients with malignant disease. However, many clinicians are uncomfortable with this approach for COPD, where opioids remain underused and barriers to opioid prescribing have been identified. ${ }^{6}$ The Canadian Thoracic Society recently released a clinical practice guideline ${ }^{7}$ that recommends the use of opioids for refractory dyspnea in patients with advanced COPD. Nevertheless, uptake of these recommendations may be limited unless long-held biases are overcome. ${ }^{6}$ To increase the understanding of these issues, we built on earlier survey work ${ }^{8}$ and a preliminary qualitative inquiry ${ }^{9}$ by conducting two studies: one to explore the experiences of patients and their family caregivers when opioids are prescribed to treat refractory dyspnea, and one to explore physicians' perspectives and attitudes toward opioid use for dyspnea in advanced COPD.

\section{Methods}

\section{Approach}

Both studies were approved by the research ethics board at Capital Health in Halifax, Nova Scotia. The physician study was approved by five other research ethics boards of the district 
health authorities in Nova Scotia. Given the dearth of evidence about patient and family caregiver experiences with opioids for dyspnea, it was appropriate to use a qualitative approach. We supplemented this approach with quantitative measures to further describe participants and maximize comprehensiveness,${ }^{10}$ an approach consistent with current calls for mixed-methods approaches to evaluate dyspnea in advanced disease ${ }^{11-13}$ and the benefit of palliative care interventions. ${ }^{13}$ To identify physicians' attitudes about and experience with prescribing opioids in this context, we used a single qualitative approach and collected basic demographic information.

\section{Setting}

The studies were conducted in both urban and rural settings. Most interviews with patients and family caregivers were conducted in their homes. (Most patients were housebound as a result of their advanced disease.) Physicians were interviewed in their offices or at a location of their choice.

\section{Sampling}

For both studies, we used purposive sampling ${ }^{14}$ to maximize the likelihood of recruiting participants with sufficient experience with the phenomenon of interest. For the study involving patients and family caregivers, the phenomenon of interest was their experiences of living with opioid therapy.

We recruited potential participants for the patient and family caregiver study from among patients with severe, stable COPD (as defined by the Canadian Thoracic Society) who had been previously prescribed and were already taking opioids for dyspnea and who were followed (currently or previously) by the Division of Respirology or by the home outreach service of the Division of Palliative Medicine at Capital Health in Halifax. We also recruited from among the family caregivers living with these patients and bereaved caregivers willing to reflect on their prebereavement experience of decedents using opioids for refractory dyspnea. Clinicians in the immediate circle of care made potential participants aware of the study. Interested patients and/or family caregivers were sent study information. Consent forms were mailed to some participants in advance; most were willing to review documentation in person, and all agreed to participate at this stage. The full inclusion and exclusion criteria are listed in Appendix 1 (www.cmaj.ca/lookup/suppl/doi:10.1503 /cmaj.111758/-/DC1).

Physicians in Nova Scotia were eligible to participate if they practised primarily in respirol- ogy, family medicine, internal medicine or palliative medicine. Within a purposive sampling framework, we used a convenience sample approach to contact potential physicians (via their office secretaries) who were likely to care for patients with COPD. Study information was sent to interested physicians, who then chose an interview time and location.

\section{Data collection and analysis}

After obtaining informed consent, we collected demographic data from patients, family caregivers and physicians (Appendix 2 and 3, www.cmaj.ca/lookup/suppl/doi:10.1503/cmaj

$.111758 /-/ D C 1)$. Two of the authors (J.Y. and C.S.) then conducted semistructured one-on-one interviews with patients, family caregivers and physicians separately until theoretical saturation was achieved. Theoretical saturation was determined by a consensus decision by the interviewers (J.Y. and C.S.) to interview no more participants at the point at which they were hearing no new information, with confirmation by the study analyst (M.D.) that no new concepts were emerging and that further interviews would not add substantively to the content.

We explored the experience of living with COPD and dyspnea (patients and family caregivers), treating patients with COPD and managing dyspnea (physicians) and attitudes about opioid use (all participants). We developed interview guides (Appendix 4 and 5, www.cmaj.ca/lookup /suppl/doi:10.1503/cmaj.111758/-/DC1) through an iterative process based on themes identified in our previous research, literature reviews conducted in preparation for these studies, and testing of the interview guide in a pilot study. ${ }^{9}$ Prompts were rarely needed because the patients and family caregivers were eager to discuss their experiences and concerns. Interviews with physicians proceeded similarly.

Patients and family caregivers completed validated quantitative assessments for the severity of dyspnea, ${ }^{11,15}$ functional status (palliative performance scale), ${ }^{16}$ anxiety and depression ${ }^{17}$ and quality of life..$^{18,19}$ Additionally, they indicated the "helpfulness" of opioids on a 5-point Likert scale $(1=$ not helpful at all; $5=$ very helpful $)$ and whether, when balancing the benefits and adverse effects, they wanted to continue opioid therapy (yes or no), and the reason(s) for their choice in terms of changes in dyspnea, quality of life and adverse effects. ${ }^{20}$ Quantitative tools were completed by patients and family caregivers following their qualitative interviews. If the participants were fatigued, they completed the tools within a few days and returned the documents to the study coordinator. 
The digitally audiorecorded interviews were transcribed verbatim. The study analyst (M.D.) used an interpretive description approach to analyze the data from each study separately. The first step in the analysis process was to describe the phenomenon of interest (the experiences of patients and family caregivers with opioid treatment for dyspnea in advanced COPD, and the perspectives of physicians caring for such patients and their attitudes about the use of lowdose opioids for the treatment of dyspnea). This was followed by an interpretive step involving all members of the team, which involved grappling with possible meanings of the described observations from a clinical perspective. The value of this methodology lies in its capacity to capture practice-relevant particularities of individual experiences as well as broader commonalities across those experiences. ${ }^{21,22}$ In addition, M.F. independently listened to early audio files (to ensure unbiased conduct of interviews), read subsequent transcriptions and reviewed the derived themes.

\section{Results}

\section{Patients' perspectives}

Of the 10 patients invited to participate, one provided consent but died before data collection. Another patient chose not to participate but her caregivers were enrolled. Eight patients were enrolled in the study (five men and three women, mean age 71 [standard deviation 9] years, range 52-79 years). Six of the eight patients lived in an urban area. Scores on the palliative performance scale ranged from $50 \%-70 \%$. All patients had a score of 5 on the Medical Research Council dyspnea scale (i.e., they were too short of breath to leave their homes or were breathless when dressing or undressing), varied in scores for dyspnea intensity, anxiety and depression, were on optimal conventional inhaled COPD therapies as per Canadian Thoracic Society guidelines, ${ }^{25}$ were using long-term oxygen therapy, and had been using opioids for dyspnea for five weeks to four years.

At the time of the interviews, three patients were taking immediate-release morphine sulfate (dose range $0.5-2 \mathrm{mg}$ four times a day), four patients were taking a combination of a sustained-released product (dose range $20-30 \mathrm{mg}$ morphine equivalent, twice a day) with additional immediate-release morphine sulfate for breakthrough dyspnea, and one patient was taking sublingual fentanyl. Commonly reported adverse effects included constipation and dry mouth. These adverse effects were usually mild, although four patients reported severe individual symptoms including dry mouth, itching, sweating and decreased libido.

All patients reported that opioids provided substantial improvements to their quality of life, relief of their dyspnea, or both, and cited this as their main reason to continue taking opioids over the longer term (Box 1). Patient responses to the quality-of-life questionnaire ${ }^{18}$ supported this finding, with five of seven patients who responded recording scores of 5 or higher (out of 10). Patients reported that opioids were either "very" or "somewhat" helpful and, balancing the benefits and adverse effects, all wished to continue taking opioids.

\section{Family caregivers' perspectives}

Family caregivers (age range 34-75 years, five men, seven women) perceived the benefits of opioids to be meaningful and substantial, and they felt that the adverse effects were not a major concern. Of the 11 caregivers who responded to our questionnaire, all supported continued opioid use, and 9 rated opioids as "very" helpful. Caregivers described opioids as helping patients to breathe more "normally"; most observed improvements in their family members' symptoms of anxiety and depression. Some described patients as looking younger, and we heard frequent comments about increased activities and ability to do more. During the interviews, many caregivers reported reductions in their own stress levels and improvements in their own quality of life (Box 2).

\section{Relationships (according to patients and family caregivers)}

We have previously described the burdens that advanced COPD imposes on relationships between spouses and/or between patients and their children. ${ }^{24,26}$ In the current study, we again heard repeated descriptions of guilt (e.g., if caregivers left patients on their own for any length of time). We also heard of resilience and a determination to "see things through" (Box 3). When

\section{Box 1: Patients' perspectives on relief of symptoms with the use of} opioids

Interviewer: How would you say the morphine has changed your breathing?

- "Ah, it takes away a lot of the struggle, you know. You're not struggling to catch your next breath. You're more relaxed ... I still lose my breath mind you if I overexert, and it doesn't take much to overexert you know, just, but ah, it's made my life more comfortable, yeah." - Patient 9

- "Well it [opioid treatment] seemed to give me a whole new outlook. Even though I didn't do much walking around and that kind of stuff, I could still do more because I felt better, oh just all over felt better ... I think it relaxes me, and I don't know how but it helps with the breathing so, if that's just because I'm relaxed or if it actually does something I don't know, which in turn makes everything go better, smoother." - Patient 1

- "If I [the patient demonstrated heavy breathing] like that, I know my oxygen and my puffer don't work so I take that [opioid] then, as soon as I take that then, three, four minutes, she's cleared right up." - Patient 5 
exploring with patients and family caregivers whether or how opioids (for dyspnea) had changed their relationships, some caregivers described how the patients' use of opioids had imposed a new routine, depending on the medication used and the setting, and that it did so within a sense of teamwork. We also heard concern that stopping morphine would shorten life (Box 3).

\section{Fears and communication}

Both patients and family caregivers expressed some concern about addiction, respiratory suppression and imminent death, but they indicated that these fears were easily and quickly alleviated by honest and open communication with their health care professionals (Box 4).

\section{Physicians' perspectives}

Twenty-eight physicians (17 men, 11 women) from urban $(n=17)$ and rural $(n=11)$ settings were enrolled from five of nine district health

\section{Box 2: Experiences and perceptions of family caregivers about the} effects of opioids

- "This is a miracle, I mean for me it's ... the grey hair I've created before fentanyl. I haven't had any more since." - Family Caregiver 2

- "Well she's not worried. She's not breaking down and crying. She's happier. She watches her game show. The look on her face is much, much better and I see her in the mornings when she comes out here to have her tea she looks great to me in comparison to what it was. Yeah, it's unreal. And if I miss, if I'm not on the ball like to get her a drink, she'll say I need my morphine and I just go in and give her that little 0.5." — Family Caregiver 9

- "... You know it's given Mom a better quality of life. It's given us so we're not so worried, watching her struggle right." - Family Caregiver 10

- "Even for when he's just like you know going out to come upstairs to go for a drive with us, you know, he knows that he has to take the liquid stuff [opioid] about 15-20 minutes before he's coming up and it makes a difference. The days that ... he doesn't take it, he's winded. So, it makes a difference. You can see it." - Family Caregiver 7

Box 3: Perspectives of patients and family caregivers on relationships, resilience and routines after opioids were started

- "I never done much cooking or anything like that but she helps me. You learn. Wash the clothes and stuff like that, I never had to. She done all that. I mixed some clothes up when I first did it and I got yelled at for that. Then I figured it out ... Yeah, you've got to do what you've got to do. Yeah, you can't be down about it. You've got to be up as high as you can. I don't dwell a lot on the end and stuff, I don't sit around." - Family Caregiver 1

- "I used to worry and worry and worry and now I just take one day at a time. Most of the time, there are some days when you think about it and then you say well look that's enough thinking. Just go and do something else. That's what I have to do, and with the grandchildren they don't give you time to think about anything anyway. Yeah, come on grammy, come on, come now and they're just so cute." - Patient 1

- "As soon as he wakes up I give him his morphine, the short-acting before he has his shower because it helps; and then as soon as he's out here, he gets his, he takes $40 \mathrm{mg}$ in 24 hours, yeah 24 hours of morphine. But I mean I think he's doing real good. I would never stop the morphine though. No. Because I don't think he'd last." - Family Caregiver 3 authorities in Nova Scotia. Upon initial contact, one physician declined participation and another declined audiorecording but agreed to notetaking (citing concerns about a tendency to some profanity at times). Physicians practised family medicine $(n=10)$, respiratory medicine $(n=6)$, internal medicine $(n=6)$ and palliative medicine $(n=6)$. Of the 25 physicians who reported their age, the median age was 52 (range 29-70) years, with a median practice experience of 20 (range 3-45) years. Many physicians expressed frustration with suboptimal control of dyspnea in patients with advancing COPD, and they expressed a desire to learn about new potential treatments. They expressed various levels of comfort with prescribing and administering opioids in hospital settings; most were uncomfortable with prescribing opioids in home or community settings. While most physicians were reluctant to prescribe opioids for patients with advanced COPD, those with experience prescribing opioids for dyspnea found them to be effective for providing some relief for some patients (Box 5). Of those who had not prescribed opioids in this context, some felt that opioids might be of value and that palliative interventions such as opioids might not be used often enough for these patients (Box 5).

The risk of respiratory depression was a concern for many physicians, including those from palliative care, although this group was more likely to prescribe opioids for COPD for patients whose symptom burden was high and poorly relieved by other conventional measures (Box 6).

Physicians from all disciplines suggested that lack of education and knowledge about opioids were the greatest barriers to opioid use in advanced COPD. They also worried about possible censure for prescribing opioids in this context. Distinctions were made between prescribing for cancer (clear guidelines available) and prescribing for dyspnea in advanced COPD, for which many felt that opioids are not seen as "standard of care." Some physicians discussed the challenges that come with prescribing opioids for chronic pain and felt that prescribing opioids for refractory dyspnea might yield similar clinical conundrums and bring increased scrutiny from drug monitoring boards or regulatory bodies (Box 7).

\section{Interpretation}

Patients reported that the benefits of opioid therapy outweighed the burdens and chose to continue to take opioids when they balanced the benefits with the adverse effects. All found opioids helpful with improvements to their quality 
of life or dyspnea or both. Concerns about safety or stigma regarding opioid treatment were rare. Family caregivers echoed this view, and some felt that opioids had actually helped to extend patients' lives. Both patients and family caregivers described the burden of living with severe, persistent dyspnea and how opioids had improved the quality of their lives. Conversely, many physicians indicated uncertainty and discomfort about prescribing opioids to patients with COPD. Lack of guidance, confidence and experience, a fear of respiratory suppression, and concern about censure were key factors limiting their willingness to prescribe opioids in this context. However, most acknowledged that dyspnea is difficult and frustrating to manage and thus were willing to consider opioids for this purpose. These findings suggest a substantial gap between patients' and caregivers' experiences and physicians' current practices related to opioid therapy for refractory dyspnea in advanced COPD.

Despite the trend toward more explicit recommendations and clinical practice guidelines from professional societies for the use of opioids to treat refractory dyspnea in patients with advanced COPD, ${ }^{7,27,28}$ many physicians remain hesitant to do so. From a medical perspective, a shroud of uncertainty and disquiet continues to surround the use and prescription of opioids for patients with COPD. Arguably, disproportionate emphasis has been placed on the risk of respiratory depression that is not supported by currently available evidence. Deep-rooted fears that militate against the use of opioids are likely based on innumerable factors, but our findings suggest that, while physicians report prognostic uncertainty, fear of hastening death via respiratory suppression, and fears of recrimination, their patients, for the most part, appear to be open to whatever will relieve their dyspnea.

We are unaware of similar studies specifically dealing with attitudes about the use of opioids for dyspnea in patients with COPD, other than our preliminary work. Our initial surveys at continuing medical education events highlighted clinicians' discomfort with prescribing opioids as a treatment for dyspnea in patients with advanced COPD. ${ }^{8}$ We followed this survey with a pilot study of respiratory therapists' and family physicians' experiences and attitudes in New Brunswick. ${ }^{9}$ In the present study, we extend this pilot work by providing insights into patients' and family caregivers' experiences with opioids, and contrasting this with physicians' perspectives and current practices.

Our interpretation of data from our two studies was inevitably influenced by the investigators' professional backgrounds (medical: G.R.;
Box 4: Perspectives of patients about fears and communication about the use of opioids

- "He [the doctor] said if I would be willing to try it, he would start it before I left the hospital to see if there would be any side effects that would really carry on after I got home. So he did. It wasn't a big deal because he explained what it was for and what was going to happen. I never thought much more of it than that. He was prescribing this to help." Q: So you weren't afraid of morphine? A: "No 'cause he explained himself, I knew what to expect and what it was for." - Patient 1

- Q: When the doctor first talked about having fentanyl, did you know that fentanyl is in the same family as morphine drugs? A: "Yes I did, because [the doctor] said something about morphine and he said, you know, l'd like to try fentanyl first. So I knew that it was associated with morphine and my preference was the fentanyl. I remember saying I don't care what it is. If it helps me I want it." Q: And if they had said it was morphine? A: "I'd still do the same thing." - Patient 2

- Q: So you said the morphine was started for your breathing and for anxiety, do you remember who started that and what was the setting? A: "Yes, she was a resident, and she and a couple of doctors said 'we're starting you on morphine.' And I said 'you're starting me on morphine, I'm scared to death of morphine.' She said it lowers your breathing. It will be in such a small bit that there's no fear of getting too attached to it, and that was my fear. If, my god, imagine in that state. But anyway, having gone through nursing, there were things in the morphine and all these narcotics, I was scared to death of them." Q: So after she explained it and explained the dose you were settled enough with that? A: "Yes, I was. It was a small enough dosage." - Patient 4

- Q: Do you remember when it was suggested the first time that you should take morphine for your breathing? A: "Yes, I do very well. I said anything! [The doctor] said sometimes we find that people get relief, breathing relief, when they take morphine. I said, oh well, it's worth a try. So she said well okay, we'll try it." - Patient 6

Box 5: Experiences and expectations of physicians about prescribing opioids for refractory dypsnea in patients with advanced COPD

Interviewer: Has opioid therapy been successful ... a helpful therapy for patients?

- "I'd say absolutely. I don't think I've ever had a significant respiratory depression that I'm aware of ... I think it's a horrible sensation to be suffocating and I really think that if you can give somebody at that stage, hopefully you've had a lot of conversations with people and one of them that always comes up is 'I'm not giving you morphine because I think you're going to die, I'm giving you morphine so that while you're living you feel good.'" - Physician 21, Family Medicine

- "We actually had a patient who was using fentanyl sublingually for their COPD. This was going on about two weeks ago and got wonderful symptomatic control from it when she did run into a spell of breathlessness ... For the majority of the time that was wonderful for her. It was just that short-acting burst of medication that worked wonderfully for her." - Physician 17, Palliative Medicine

- "I think it's more than a placebo. I really do ... I have an interest in dyspnea and I know that it can be modified by a number of different factors when it gets to the brain and my thinking is that that's where morphine works, is to modify that sensation, and so I think it's more than a placebo. I'm still hoping as to whether it really works, but I believe it should." - Physician 4, Respirology

- "I think it's a great idea but I haven't read the data ... I've never, never done it but it makes good sense. We do it in acute settings. We give morphine nebules or morphine intravenous when patients are acutely dyspneic. I don't know why it's not used more often, but I hardly ever see it." - Physician 8, Internal Medicine

Note: $C O P D=$ chronic obstructive pulmonary disease. 
respiratory therapy: J.Y.; psychospiritual: C.S. and M.D.). All authors have conducted and completed prior qualitative studies in either clinical contexts of COPD or dyspnea $a^{9,23,24}$ or as part of doctoral or master's thesis work (C.S., M.D., M.F.).

Congestive heart failure is similar to COPD in that patients tend to be older, have major comorbidity and, in later stages of illness, often experi-

\section{Box 6: Physicians' concerns about prescribing opioids for refractory} dypsnea in patients with advanced COPD

- "I mean when I went to medical school, we were taught to never consider opioids in people with COPD. We were going to kill them. It was like if you gave them one dose of morphine, they would be dead and it would be your fault. That was the teaching. So it was a revelation to me, I don't know, five, seven years ago to go to a CME thing and have people talking about using opioids and starting to use it, and certainly in terms of dealing with anxiety and breathlessness." - Physician 13, Family Medicine

- "In my generation of physicians when you were in med school that was hammered in, 'Well you can't do this. You're going to have respiratory depression.' I'm sure there is a 10-year generation where that was hammered into you, just like, 'Don't use a beta-blocker in heart failure.' So you have to relearn. I think it's just a matter of re-learning and being comfortable using it. - Physician 21, Family Medicine

- "So, I'm not concerned with respiratory depression if a patient is started on an appropriate low dose, titrated appropriately, and monitored appropriately." - Physician 3, Palliative Medicine

- "I worry about it all the time. People overdose, old and frail people really easily, when they [other health professionals] are not used to giving opioids. So they hear about giving opioids for shortness of breath and they lay it on to them and even the people who have pain, that scares me if they're people that don't understand that $5 \mathrm{mg}$ of morphine is not the same as $5 \mathrm{mg}$ of Dilaudid or, you know, they don't know how to convert to the fentanyl patch. So I worry about it all the time when other people are doing it. I don't worry about when I do it, no."

- Physician 11, Palliative Medicine

Note: $\mathrm{CME}=$ continuing medical education, $\mathrm{COPD}=$ chronic obstructive pulmonary disease

Box 7: Perspectives of physicians about regulatory bodies and prescribing opioids for refractory dypsnea in patients with advanced COPD

- "I must admit, I would be leery even at this point, on this date in February 2010, to introduce a narcotic. It would be a hard sell. There has to be some leading edge in this. There will be a delay in using it and there will be people who will go to their deaths that perhaps could have received better comfort. So the long and short of it is, I think, that I would love to start using it. If I start using it, it will come under criticism. That's how blind we are nowadays. And it will be under review. So, it's a sad situation." - Physician 22, Family Medicine

- "Well part of the problem is they've been so taught that if they give any sedatives for bad lung patients they will kill them. You have to get rid of that kind of longstanding teaching. They're frightened to death that they're going to give someone a shot of narcotic or a shot of benzo and the patient will stop breathing immediately and they'll be charged with murder." - Physician 18, Internal Medicine

- "I think, too, you're not supported. ... you have a family member that's really quite apprehensive, you really do set yourself up for the legality of it and I think that's what people are afraid of. Instead of looking in terms of the ethical side of it, perhaps. Is it ethical to not give somebody something you believe can relieve their suffering? That's a good question." - Physician 21, Family Medicine

Note: COPD = chronic obstructive pulmonary disease. ence refractory dyspnea or cough or both. Using a similar methodologic approach, Oxberry and colleagues in the United Kingdom interviewed 10 patients with severe congestive heart failure about their attitudes toward the use of morphine as a treatment for severe dyspnea or cough. ${ }^{29}$ They also found a rather surprising openness by participants to trying opioids for this purpose. Two patients in their study suggested that morphine "could be used to prolong or preserve life," 29 similar to our caregivers' suggestions that morphine may have extended the life of patients with COPD. Although their study did not directly address physicians' attitudes, some participants commented on their physicians' negative views of using opioids except as a last resort, which mirrored some of our findings. The authors stated that "patients clearly sense when their doctor has reservations about a treatment, and as trust in the prescriber was important in their willingness to try morphine, measures that can help clinicians prescribe appropriately with confidence should be encouraged." 29 This echoes our conclusions about the need for further research into physicians' concerns and approaches to education and evidence-based guidelines that can address these concerns.

Recent qualitative work involving physicians from the United States that focused on attitudes surrounding opioid prescribing for pain at the end of life ${ }^{30}$ or for noncancer pain among elderly adults $^{31}$ also reported fear of censure (via drug enforcement agencies) and the need to improve knowledge, as well as overcome barriers to and fears surrounding opioid prescribing. ${ }^{30}$ There is little data on real-time prescribing of opioids for COPD-related dyspnea. Retrospective data from a Saskatchewan database indicate a far lower prevalence of opioid prescribing toward the end of life among patients with COPD who have dyspnea than among patients with lung cancer. ${ }^{32}$ The strongest evidence available at this time from clinical trials (a systematic review favouring the use of opioids ${ }^{33}$ and a short-term randomized crossover trial $^{34}$ ) supports the experiences of the patients and family caregivers interviewed in our study (i.e., effective relief of dyspnea without evidence of respiratory depression). More recently, in a dose-increment and pharmacovigilance study involving 65 patients in Australia in which opioids were used for refractory dyspnea (mostly due to COPD), ${ }^{35}$ there were no cases of respiratory depression during a mean follow up of three months, and $51 \%$ of patients reported a benefit sufficient for them to continue taking opioids. ${ }^{35}$ Preliminary data from an ongoing Canadian clinical trial (clinical trials.gov, trial no. NCT00982891) indicate even higher levels of 
self-reported efficacy and acceptability over a period of two to six months. ${ }^{36}$

\section{Limitations}

The aim of qualitative studies is not generalizability, and our study is no exception. However, by conducting our study in both urban and rural settings, we hoped to generate new insight applicable to both. We accept that our quantitative data from the included patients and family caregivers (collected once and a small sample) was too limited to be generalizable; we have thus not reported all of the details here. Although we recruited physicians from several disciplines and from urban and rural settings, the views of the participating physicians may not represent the views of all those who care for patients with advanced COPD in Nova Scotia or elsewhere in Canada.

We recognize a potential bias in that we interviewed only patients who were taking opioids, and so we cannot report the views or concerns of patients or family caregivers who had been offered and declined or who had discontinued the use of opioids; however, we were primarily interested in those who had used opioids. We chose to enrol family caregivers who were living with patients who used opioids; the experiences of family caregivers who live elsewhere may be different. We are unable to comment on whether anxiety or depression had improved substantially for patients after opioids were started, but caregivers often mentioned that their loved one appeared "more relaxed." We did not collect spirometric measures of airflow obstruction (i.e., $\mathrm{FEV}_{1}$ or $\mathrm{FEV}_{1} / \mathrm{FVC}$ ratio); most of the included patients were housebound, living with high dyspnea burden and had not undergone recent pulmonary function testing.

\section{Conclusion}

If the perspectives of the physicians interviewed represent those of physicians who practise elsewhere in Canada, patients living with advanced COPD and refractory dyspnea are unlikely to experience treatment benefits comparable to those of our study participants. Family members may also be denied potential relief of associated stress that can burden them for years. ${ }^{24}$ Discrepancies between the positive experiences of patients and family caregivers and the reluctance of physicians to prescribe opioids for refractory dyspnea constitute an important gap in care. Bridging this gap will likely require innovative educational initiatives to improve the uptake of guidelines and confidence in prescribing opioids for refractory dyspnea. At professional society conferences in both Canada and the United States, we have used, to good effect, film footage of patients and their caregivers discussing the effects of opioids for refractory dyspnea. Others have recently promoted the concept of a rapid learning model, similar to the "plan, do, study, act" cycle promoted by the Institute for Healthcare Improvement, ${ }^{37}$ to disseminate new information from clinical trials and consensus statements from professional societies about palliation of dyspnea. ${ }^{38}$

To address the many knowledge gaps regarding patient and caregiver perspectives, we are collecting, in an ongoing clinical trial, both quantitative and qualitative assessments of patient and caregiver experiences before and for six months after opioids are initiated for refractory dyspnea in advanced COPD.

Our patients and caregivers have taught us much about effective palliation; we have a duty to act on their insights. The Canadian Medical Association's code of ethics reminds us of our responsibility to "first consider the wellbeing of the patient" and that doing so requires our engagement in "lifelong learning to maintain and improve ... professional knowledge, skills, and attitudes. ${ }^{\prime 39}$ Evidence is accumulating to suggest that soon the appropriate question will no longer be if we should prescribe opioids to help palliate refractory dyspnea in patients living with advanced COPD, but rather how to do this competently $y^{7,40}$ and when.

\section{References}

1. Gershon AS, Wang C, Wilton AS, et al. Trends in chronic obstructive pulmonary disease prevalence, incidence, and mortality in Ontario, Canada, 1996 to 2007: a population-based study. Arch Intern Med 2010;170:560-5.

2. Elkington $\mathrm{H}$, White $\mathrm{P}$, Addington-Hall $\mathrm{J}$, et al. The healthcare needs of chronic obstructive pulmonary disease patients in the last year of life. Palliat Med 2005;19:485-91.

3. Rocker GM, Dodek PM, Heyland DK. Toward optimal end-oflife care for patients with advanced chronic obstructive pulmonary disease: Insights from a multicentre study. Can Respir J 2008; 15:249-54.

4. Heyland DK, Dodek P, Rocker G, et al. What matters most in end-of-life care: perceptions of seriously ill patients and their family members. CMAJ 2006;174:627-33.

5. Heyland DK, Cook DJ, Rocker GM, et al. Defining priorities for improving end-of-life care in Canada. CMAJ 2010;182:E747-52.

6. Rocker G, Horton R, Currow D, et al. Palliation of dyspnoea in advanced COPD: revisiting a role for opioids. Thorax 2009;64: 910-5.

7. Marciniuk DD, Goodridge D, Hernandez P, et al.; Canadian Thoracic Society COPD Committee Dyspnea Expert Working Group. Managing dyspnea in patients with advanced chronic obstructive pulmonary disease: A Canadian Thoracic Society clinical practice guideline. Can Respir J 2011;18:69-78.

8. Rocker GM, Young J, Horton R. Using opioids to treat dyspnea in advanced COPD: A survey of Canadian clinicians. http://meeting .chestpubs.org/cgi/content/abstract/134/4/s29001. 2008. (accessed 2012 Mar. 21).

9. Young J, Donahue M, Simpson AC, et al. Attitudes to using opioids to treat dyspnea in advanced copd: a qualitative study of family physicians and respiratory therapists. Can Fam Physician. In press.

10. O'Cathain A, Murphy E, Nicholl J. Why, and how, mixed methods research is undertaken in health services research in England: a mixed methods study. BMC Health Serv Res 2007;7:85

11. Bausewein C, Farquhar M, Booth S, et al. Measurement of breathlessness in advanced disease: a systematic review. Respir Med 2007;101:399-410.

12. Rocker G. Dyspnea: recent insights and innovations. Prog Palliat Care. 2011;19:219-22. 
13. Farquhar MC, Ewing G, Booth S. Using mixed methods to develop and evaluate complex interventions in palliative care research. Palliat Med 2011;25:748-57.

14. Strauss A, Corbin J. Basics of qualitative research. techniques and procedures for developing grounded theory. 2 nd ed. London (UK): Sage publications; 1998.

15. Puhan MA, Guyatt GH, Goldstein R, et al. Relative responsiveness of the Chronic Respiratory Questionnaire, St. Georges Respiratory Questionnaire and four other health-related quality of life instruments for patients with chronic lung disease. Respir Med 2007;101:308-16.

16. Anderson F, Downing GM, Hill J, et al. Palliative performance scale (PPS): a new tool. J Palliat Care 1996;12:5-11.

17. Dowson C, Laing R, Barraclough R, et al. The use of the Hospital Anxiety and Depression Scale (HADS) in patients with chronic obstructive pulmonary disease: a pilot study. $N \mathrm{Z} \mathrm{Med} \mathrm{J}$ 2001;114:447-9.

18. Cohen SR, Mount BM, Strobel MG, et al. The McGill Quality of Life Questionnaire: a measure of quality of life appropriate for people with advanced disease. A preliminary study of validity and acceptability. Palliat Med 1995;9:207-19.

19. Cohen R, Leis AM, Kuhl D, et al. QOLLTI-F: measuring family carer quality of life. Palliat Med 2006;20:755-67.

20. Smith HS. The Numerical Opioid Side Effect (NOSE) assessment tool. J Cancer Pain Symptom Palliation 2006;1:3-6.

21. Thorne S, Con A, McGuinness L, et al. Health care communication issues in multiple sclerosis: an interpretive description. Qual Health Res 2004; 14:5-22.

22. Thorne S. Interpretive description. Walnut Creek (CA): Left Coast Press Inc.; 2008. p. 272.

23. Booth S, Farquhar M, Gysels M, et al. The impact of a breathlessness intervention service (BIS) on the lives of patients with intractable dyspnea:A qualitative phase 1 study. Palliat Support Care 2006;4:287-93.

24. Simpson AC, Young J, Donahue M, et al. A day at a time: caregiving on the edge in advanced COPD. Int J Chron Obstruct Pulmon Dis 2010;5:141-51.

25. O'Donnell DE, Hernandez P, Kaplan A, et al. Canadian Thoracic Society recommendations for management of chronic obstructive pulmonary disease - 2008 update — highlights for primary care. Can Respir J 2008;15(Suppl A):1A-8A.

26. Simpson AC, Rocker GM. Advanced chronic obstructive pulmonary disease: impact on informal caregivers. J Palliat Care 2008;24:49-54.

27. Lanken PN, Terry PB, Delisser HM, et al. An official American Thoracic Society clinical policy statement: palliative care for patients with respiratory diseases and critical illnesses. Am J Respir Crit Care Med 2008;177:912-27.

28. Mahler DA, Selecky PA, Harrod CG, et al. American College of Chest Physicians consensus statement on the management of dyspnea in patients with advanced lung or heart disease. Chest 2010;137:674-91.

29. Oxberry SG, Jones L, Clark AL, et al. Attitudes to morphine in chronic heart failure patients. BMJ Support Palliat Care 2011 Dec. 13 [epub ahead of print].

30. Zerzan J, Lee CA, Haverhals LM, et al. Exploring physician decisions about end- of-life opiate prescribing: a qualitative study. J Palliat Med 2011;14:567-72.

31. Spitz A, Moore AA, Papaleontiou M, et al. Primary care providers' perspective on prescribing opioids to older adults with chronic non-cancer pain: a qualitative study. BMC Geriatr 2011;11:35.
32. Goodridge D, Lawson J, Rocker G, et al. Factors associated with opioid dispensation for patients with COPD and lung cancer in the last year of life: A retrospective analysis. Int J Chron Obstruct Pulmon Dis 2010;5:99-105.

33. Jennings AL, Davies AN, Higgins JP, et al. A systematic review of the use of opioids in the management of dyspnoea. Thorax 2002;57:939-44.

34. Abernethy AP, Currow DC, Frith P, et al. Randomised, double blind, placebo controlled crossover trial of sustained release morphine for the management of refractory dyspnoea. BMJ 2003;327:523-8.

35. Currow DC, McDonald C, Oaten S, et al. Once-daily opioids for chronic dyspnea: a dose increment and pharmacovigilance study. J Pain Symptom Manage 2011;42:388-99.

36. Rocker G, Demmons J, Donahue M, et al. Using opioids to treat refractory dyspnea in advanced COPD: preliminary insights from a clinical trial. Chest 2011;140:543A. Available: http://chestjournal.chestpubs.org/cgi/content/meeting_abstract/1 40/4_MeetingAbstracts/543A (accessed 2012 Mar. 26).

37. Lynn J, Nolan K, Kabcenell A, et al. Reforming care for persons near the end of life: the promise of quality improvement. Ann Intern Med 2002;137:117-22.

38. Kamal AH, Miriovsky BJ, Currow DC, et al. Improving the management of dyspnea in the community using rapid learning approaches. Chron Respir Dis. In press.

39. Code of ethics of the Canadian Medical Association. CMAJ 1996;155:1063-5.

40. Gallagher R. Killing the symptom without killing the patient. Can Fam Physician 2010;56(6):544-546, e210-512.

Affiliations: From the Division of Respirology (Rocker, Young, Donahue, Simpson), Queen Elizabeth II Health Science Centre and Dalhousie University, Halifax, NS; and the General Practice and Primary Care Research Unit (Farquhar), Department of Public Health and Primary Care, University of Cambridge, Cambridge, UK.

Contributors: Graeme Rocker, Joanne Young and Catherine Simpson contributed to the conception and design of the study. Interviews were conducted by Joanne Young and Cathy Simpson, and verbatim transcriptions were analyzed by Margaret Donahue. All of the authors contributed to the interpretation of data, drafting and revising the article, and gave final approval of the version submitted for publication.

Funding: The study involving patients and family caregivers was unfunded. The study involving physicians was supported by a grant from the Legacy Fund of the Lung Association of Nova Scotia. The study sponsor had no role in the design of the study, collection or analysis of data, the writing of the report or the decision to submit the article for publication. Morag Farquhar is funded by a Macmillan Cancer Support Post-doctoral Fellowship.

Acknowledgements: The authors thank Dr. Robert Horton for his longstanding and insightful support of our research endeavours, and Ms. Shirley Wheaton, whose meticulous transcription of the interview audio files was essential to our analysis. 